\title{
Two laser beams interaction in photonic crystal fibers infiltrated with highly nonlinear materials
}

\author{
M. Murek, K.A. Rutkowska \\ Faculty of Physics, Warsaw University of Technology, Koszykowa 75, 00-662 Warszawa
}

Received June 22, 2014; accepted June 29, 2014; published June 30, 2014

\begin{abstract}
In this letter, the results of experimental tests on the discrete light propagation in photonic liquid crystal fibers (PLCFs) are presented. More precisely, this work is mainly inquiring into the matter how two laser beams simultaneously launched into PLCF can influence the optical properties of the fiber. For this purpose, a weak collinear probe beam at the different wavelength has been applied to monitor how the waveguide channel(s) is (are) decoupled from the rest of the matrix due to the influence of the pump laser beam. Firstly, a low-power signal beam at a wavelength of $532 \mathrm{~nm}$ was co-launched axially with the pump beam $(1064 \mathrm{~nm})$ to confirm the power-dependence of optical properties (induced by the pump) while observing the signal beam profile at the output facet of PLCF. Secondly, the beams from the same laser sources in the inverted configuration were applied.
\end{abstract}

Photonic crystal fibers (PCFs) are a special class of optical fibers with their characteristics resulting from the unique properties of photonic crystals [1-2]. While typical PCFs consist of air channels arranged periodically in a solid material (e.g. silica, multicomponent glass, polymer), they often serve as a passive host structure to be infiltrated with different gaseous and fluid materials. This infiltration allows for additional (dynamical) adjustment of the guiding properties of PCFs. Specifically, the first band-gap tuning was demonstrated in solid core PCF infiltrated with a high index fluid by Bise et al. in 2002 [3]. One year later Larsen et al. [4] infiltrated PCF with liquid crystals (LCs). The application of LCs as an active inclusion has resulted in a new type of photonic structures, often referred to as photonic liquid crystal fibers (PLCFs). High tunability of PLCFs (in terms of changing in propagation and spectral properties) arises mainly from special optical characteristics of LCs. The latter can be dynamically adjusted by changing e.g. temperature, electric field, magnetic field or beam power.

There are two main types of light guiding mechanisms in $\mathrm{P}(\mathrm{L}) \mathrm{CFs}$. The first one is called modified total internal reflection (mTIR) and occurs in a solid core PCF (Fig. 1a) or in a hollow core PCF infiltrated with LC [characterized by a refractive index higher than that of the material forming PCF structure] (Fig 1b). The second mechanism is a photonic band-gap (PBG) guiding which takes place in an air core PCF (Fig. 1c) or in holey fiber infiltrated with LC with a refractive index higher than that of the material used for PCF (Fig. 1d).
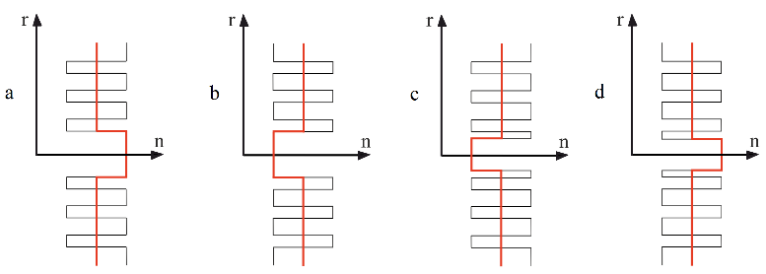

Fig. 1. Refractive index profiles (grey lines) in: a) solid core PCF, b) solid core PLCF, c) hollow core PCFs, d) hollow core PLCF.Red lines correspond to the effective refractive index of the structures.

On the other hand, PCF can be also considered as a two-dimensional photonic lattice with periodic modulation of a refractive index in two dimensions), allowing thus (in specific cases) for discrete light propagation [5-6]. Specifically, completely [7-9] and selectively [10] fluid-infiltrated PCFs have been successfully adopted to the needs of discrete optics.

In this work a hollow core PCF made of silica glass and fabricated at the Maria Curie Sklodowska University (MCSU) has been chosen as a host structure. The cross section of the structure in the form of a uniform hexagonal lattice composed of air-holes distributed in the silica cladding and arranged in six concentric rings is shown in Fig. 2. This particular geometry (with a central air-hole in the central part of the fiber cross-section) when infiltrated with a liquid crystalline material can be considered as a matrix of waveguide channels and its regularity (with no defect in the form of a solid core as it takes place in typical PCFs) has been chosen to study discrete light propagation in identical conditions to those in uniform $2 \mathrm{D}$ waveguide matrices [5-6].

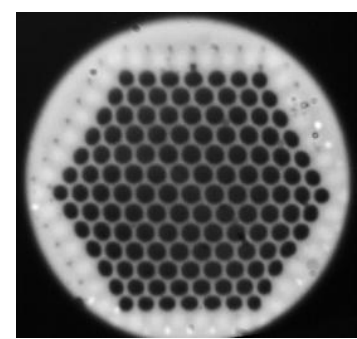

Fig.2. Photo of the host photonic crystal fiber manufactured at the MCSU, Lublin, Poland. The geometrical parameters of the fiber are as follows: the air-hole diameter $\mathrm{d}=4.4 \mu \mathrm{m}$ and the holes spacing $\Lambda=5.9 \mu \mathrm{m}$. 
Three different thermotropic nematic liquid crystals, namely: 1110, 6CHBT and E7 (all of them synthesized at the Military University of Technology, Warsaw, Poland) have been selected to serve as a guest material in PLCF. Temperature dependences of refractive indices of those LCs are shown in Fig. 3.

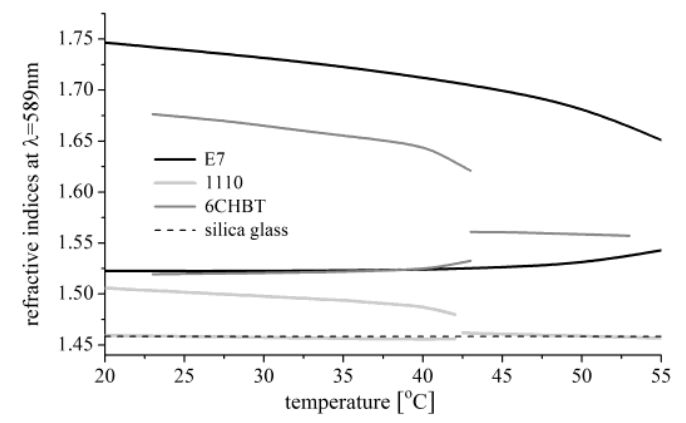

Fig. 3. Refractive indices as a function of temperature for 1110 , 6CHBT, E7 nematic LCs (all with positive birefringence, i.e. $n_{e}>n_{o}$ ) at the wavelength of $589 \mathrm{~nm}$. Temperature dependence of refractive index for the silica glass at the same wavelength is represented by the dashed black line.

1110 is a liquid crystalline mixture composed of alkyl 4-trans-(4-trans-slkylcyclohexyl) cyclohexylcarbonates. It is characterized by relatively low birefringence $(\Delta n \approx 0.05)$ and in some ranges of temperatures (close to the room one) its ordinary refractive index is lower than that for silica glass. In our experiments we have also used 4(trans-4-hexyl-cyclohexyl)-isothio-cyanatobenzene called $6 \mathrm{CHBT}$ as nematic with medium birefringence $(\Delta n \approx 0.15)$ and a well known liquid crystalline mixture E7 with relatively high birefringence $(\Delta n \approx 0.2)$ which is based on cyanobiphenyls.

The simplest method of PCFs' infiltration is to put them into a container with liquid crystalline materials. Thanks to capillary forces, LCs are drawn into the air channels and the process of infiltration is strongly dependent on the temperature of LCs (mainly due to viscosity) and on the diameter of the holes in a PCF structure.

In this work, light beam propagation in PLCFs was experimentally investigated in the setup whose scheme is presented in Fig. 4. Two lasers were used as light sources, namely $\mathrm{CW} \mathrm{Nd}$ :YAG and DPSS (based on Nd:YAG) generating radiation with wavelengths of $1064 \mathrm{~nm}$ and $532 \mathrm{~nm}$, respectively. A CCD camera (connected to the computer) was used as a detector of light intensity distribution at the input and output facet of the PLCF sample. The temperature was stabilized and tuned with the use of a Peltier cell. The power of the laser beams was monitored by a power meter.

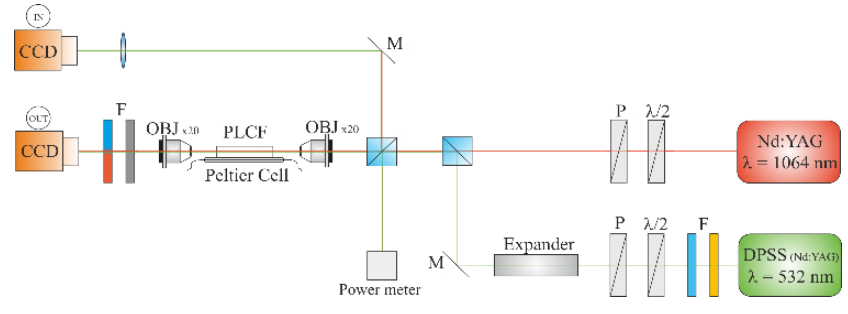

Fig. 4. Schematic drawing of the experimental setup.

Initially, a laser beam from a DPSS laser $(\lambda=532 \mathrm{~nm})$ was used as a signal while a $\mathrm{CW} \mathrm{Nd}$ :YAG laser $(\lambda=1064 \mathrm{~nm})$ served as a pump: both of them launched axially to the same liquid crystalline core in PLCF. In the second part of the experiment the role of laser sources was inverted. It has to be underlined that a set of dielectric and colored glass filters was placed directly in the front of a CCD camera. Specifically, when a green laser beam $(\lambda=532 \mathrm{~nm})$ was used as a pump, a longpass filter (with cut-on wavelength of $650 \mathrm{~nm}$ ) was applied in order to eliminate the pump beam and to register only the signal (infrared) laser beam on the CCD camera. In the inverted configuration, i.e. when an IR beam $(\lambda=1064 \mathrm{~nm})$ served as a pump, a bandpass colored glass filter (with non zero transmission for the wavelengths in the range from 355 to $610 \mathrm{~nm}$ ) was used to register the green light (without the infrared) on the CCD camera.

The signal beam was characterized by a low power in the range of parts of milliwatts (in order to avoid any nonlinear effect that could be induced by the signal beam). On the other hand the pump beam with a power of several milliwatts was used. We have checked that this power level is high enough for effects induced by thermal nonlinearity in LCs to occur.

Photos of the output facet of PLCF sample infiltrated with $1110 \mathrm{LC}$ and for a $\mathrm{CW} \mathrm{Nd}$ :YAG laser acting as a pump are shown in Figs. 5 a)-d). The length of the fiber was set as short as $0.8 \mathrm{~cm}$ because of the high light attenuation in liquid crystalline cores. The temperature of the sample was kept at $20^{\circ} \mathrm{C}$ and the power of the signal beam fixed at the constant level with its value equal to $0.014 \mathrm{~mW}$. As one can see in Fig. 5a, the green laser beam is transmitted through the sample when the pump beam is off. For a pump power of $0.36 \mathrm{~mW}$ (Fig. 5b) transmission is getting lower but then by increasing the pump power even further (to $2.36 \mathrm{~mW}$ or to $5.86 \mathrm{~mW}$ ) the light intensity of the light is getting higher (comparing to situation without the pump beam) as it is a characteristic for a thermal nonlinearity effect occurring in PLCFs [7-8, 11-13]. When PCF sample (of the length of $1.5 \mathrm{~cm}$ ) was infiltrated with 6CHBT and E7 LCs no change in propagation of signal beam induced by the pump was observed. 


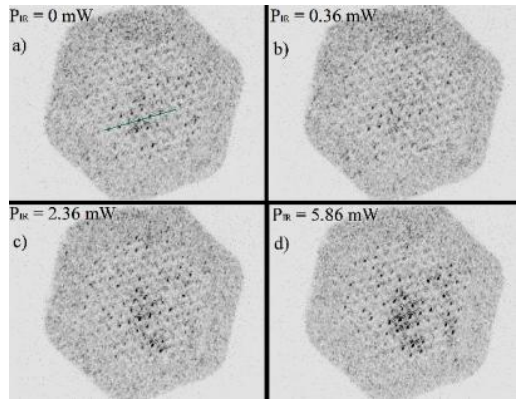

e)

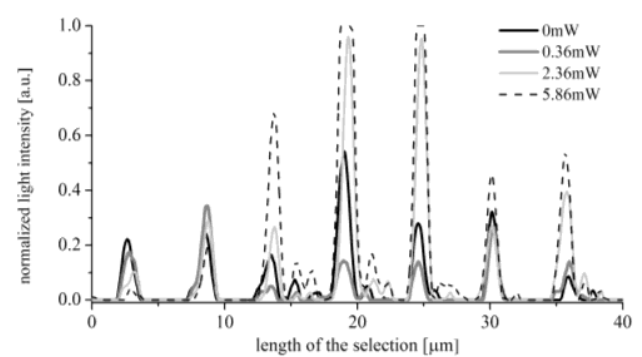

Fig. 5. Change in the output spatial profile of the signal beam $\left(\mathrm{P}_{\mathrm{G}}=0.014 \mathrm{~mW}\right)$ obtained by varying power of the pump beam $\mathrm{P}_{\mathrm{IR}}$ in PCF of interest infiltrated with 1110 LC. Panels a)-d): photos (in inverted scale of colors) of the output facet of the fiber taken with a bandpass colored glass filter blocking IR radiation; e): normalized intensity profiles for the selection of the photos taken for different values of powers of the pump beam (the latter not visible on photos).

For the laser beams used in inverted configuration, no effect of the pump beam on the signal propagation was observed for PCF infiltrated with 1110 and E7 LCs. However, when 6CHBT LC was used as an infiltration (in $\mathrm{PCF}$ of the length of $1.5 \mathrm{~cm}$ tested in temperature of $20^{\circ} \mathrm{C}$ ) the increasing in the transmission of the signal was observed growing power of the pump (see Fig. 6).

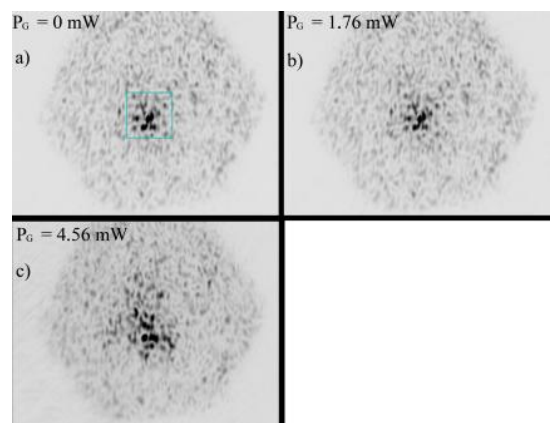

d)

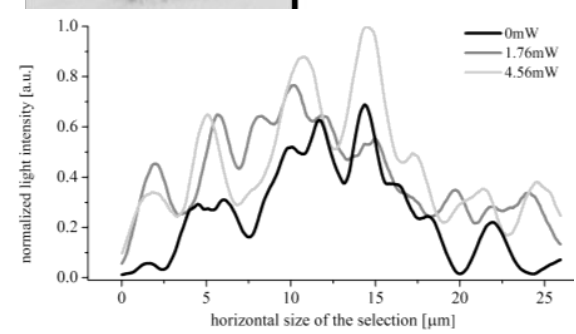

Fig. 6. Tuning of the infrared signal beam $\left(\mathrm{P}_{\mathrm{IR}}=0.15 \mathrm{~mW}\right)$ with use of the pump beam with varying power $\left(\mathrm{P}_{\mathrm{G}}\right)$ in $\mathrm{PCF}$ infiltrated with $6 \mathrm{CHBT}$ LC. Normalized intensity for the plot profiles [as selected in a)] for different powers of the infrared pump beam are shown in panel d).
In conclusion, the possibility of the change in the light intensity (and spatial profile) of the signal beam at the output facet of PCFs infiltrated with 1110 and 6CHBT LCs using the pump beam has been presented. The results prove that the propagation properties of PLCFs for low power signal beam can be obtained using variable power of the pump beam. High index contrast for PCF infiltrated with E7 LCs leads to no change in optical properties of the fiber by using even a high value of the pump laser beam power.

The authors would like to acknowledge Homing Plus Programme (HOMING PLUS/2010-2/11) granted by the Foundation for Polish Science for implementation of the project "Nonlinear photonic liquid crystal fibers". The authors would like also to thank Dr. P. Mergo (MCSU) for PCF samples and Prof. Dąbrowski (MUT) for LCs.

\section{References}

[1] P.St.J. Russell, Science 299, 358 (2003).

[2] E. Poli, A. Cucinotta, S. Selleri, Photonic Crystal Fibers. Properties and Applications (Springer, The Netherlands 2007).

[3] R.T. Bise, R.S. Windeler, K.S. Kranz, C. Kerbage, B.J. Eggleton, D.J. Trevor, Opt. Fiber Comm. Conf. Techn. Digest, 466 (2002).

[4] T.T. Larsen, A. Bjarklev, D.S. Hermann, J. Broeng, Opt. Expr. 11, 2589 (2003)

[5] I.L. Garanovich, S. Longhi, A.A. Sukhorukov, Y.S. Kivshar, Phys. Rep. 518, 1 (2012).

[6] F. Lederer, G.I. Stegeman, D.N. Christodoulides, G. Assanto, M. Segev, Y. Silberberg, Phys. Rep. 463, 1 (2008).

[7] K.A. Brzdąkiewicz, U.A. Laudyn, M.A. Karpierz, T.R. Woliński, J. Wójcik, Opto-Electron. Rev. 14, 287 (2006).

[8] U.A. Laudyn, K.A. Rutkowska, R.T. Rutkowski, M.A. Karpierz, T.R. Woliński, J. Wójcik, Centr. Europ. J. Phys. 6(3), 612 (2008).

[9] C.R. Rosberg, F.H. Bennet, D.N. Neshev, P.D. Ramussen, O. Bang, W. Krolikowski, A. Bjarklev, Y.S. Kivshar, Opt. Expr. 15(19), 12145 (2007).

[10] M. Vieweg, T. Gissibl, Y. Kartashov, L. Torner, H. Giessen, Opt. Lett. 37, 2454 (2012).

[11] K.A. Rutkowska, U.A. Laudyn, P.S. Jung, Phot. Lett. Poland 5(1), 17 (2013).

[12] K.A. Rutkowska, U.A. Laudyn, P.S. Jung, IEEE Xplore 2012, DOI: 10.1109/PGC.2012.6457983

[13] K.A. Rutkowska, U.A. Laudyn, P.S. Jung, IEEE Xplore 2013, DOI: 10.1109/CLEOE-IQEC.2013.6800904 